\title{
Value of nasal endoscopy and probing in the diagnosis and management of children with congenital epiphora
}

\author{
C J MacEwen, J D H Young, C W Barras, B Ram, P S White
}

\begin{abstract}
Background-Congenital nasolacrimal obstruction is usually the result of failure of canalisation of the distal end of the nasolacrimal duct. The most common outcome is spontaneous resolution, but some children do require surgical treatment by probing. Probing is a blind procedure with a recognised failure rate. Methods-In 52 lacrimal systems of 40 children nasal endoscopy was combined with a "stepwise" systematic probing in an attempt to improve the outcome and reduce the number of repeat procedures. Results-Combined nasal endoscopy and probing improved the understanding of outflow obstruction in young children. The success of the procedure depended upon the level of the obstruction within the outflow system. Formation of a false passage was seen in six cases (15\%). The probe was rerouted under direct visualisation in these cases to form a functioning passage. Reasons for failure were identified in those who did not have a successful outcome and only one repeat procedure was required.

Conclusion-Using nasal endoscopy the area of lacrimal outflow obstruction at the lower end of the nasolacrimal duct can be observed directly and it is possible to guide the progress of probing under direct vision. This gives better information about the nature of the obstruction, minimises the formation of false passages, and allows a wider range of treatment options under a single anaesthetic.

(Br f Ophthalmol 2001;85:314-318)
\end{abstract}

Department of

Ophthalmology, Ninewells Hospital, Dundee DD1 9SY, UK

C J MacEwen

J D H Young

C W Barras

Department of Otolaryngology

B Ram

P S White

Correspondence to: Dr MacEwen

c.j.macewen@dundee.ac.uk

Accepted for publication 13 September 2000 of the lacrimal system under general anaesthesia. Conventional probing is a blind procedure that has not changed significantly in the past
100 years. False passage formation, traumatic stenosis, and unexplained failure are recognised problems of this procedure. ${ }^{19} 20$ In addition, the high rate of spontaneous resolution provides a natural selection process in which increasingly complex problems become relatively more common as symptomatic children grow older. ${ }^{21}$

This study used a stepwise probing procedure aimed at diagnosing the level and degree of obstruction and used nasal endoscopy to directly visualise the lower end of the nasolacrimal duct during the procedure. The aims were: (1) to provide more accurate diagnosis regarding the level and degree of lacrimal outflow obstruction in children with the signs and symptoms of CNLDO; (2) to identify the prognosis regarding each type of anomaly; (3) to reduce the risk of false passage formation; and (4) to reduce the number of procedures required by any one child.

\section{Methods}

All children with CNLDO who underwent surgical intervention between June 1996 and December 1999 in our hospital were included in the study. The diagnosis was made based on a history of epiphora plus or minus discharge since birth, or shortly after birth, in one or both eyes supported by objective evidence of reduced lacrimal outflow using a fluorescein disappearance test (FDT). ${ }^{22}$

On the basis of previous studies on spontaneous resolution ${ }^{5-12}$ we have restricted surgical management to those children with signs and symptoms of CNLDO that persist after the age of 12 months. Surgery was undertaken jointly by a rhinologist, who had trained in functional endoscopic sinus surgery, and an ophthalmologist. The endoscopic view on the video monitor allowed steps in the procedure and decision making to be coordinated. The procedure was carried out under a general anaesthetic in all cases.

The surgical procedure was carried out in a stepwise manner designed to yield maximum diagnostic information. Detailed aspects of the endoscopic technique have already been described, ${ }^{23}$ but the stepwise nature of the procedure will be presented below as this illustrates the diagnostic criteria and the background to decision making made at each stage (Fig 1).

In brief, an initial nasal endoscopy was performed in order to confirm normal nasal anatomy, in particular the proximity of the inferior turbinate to the lateral nasal wall. Ocular examination was carried out to exclude 
PROBING

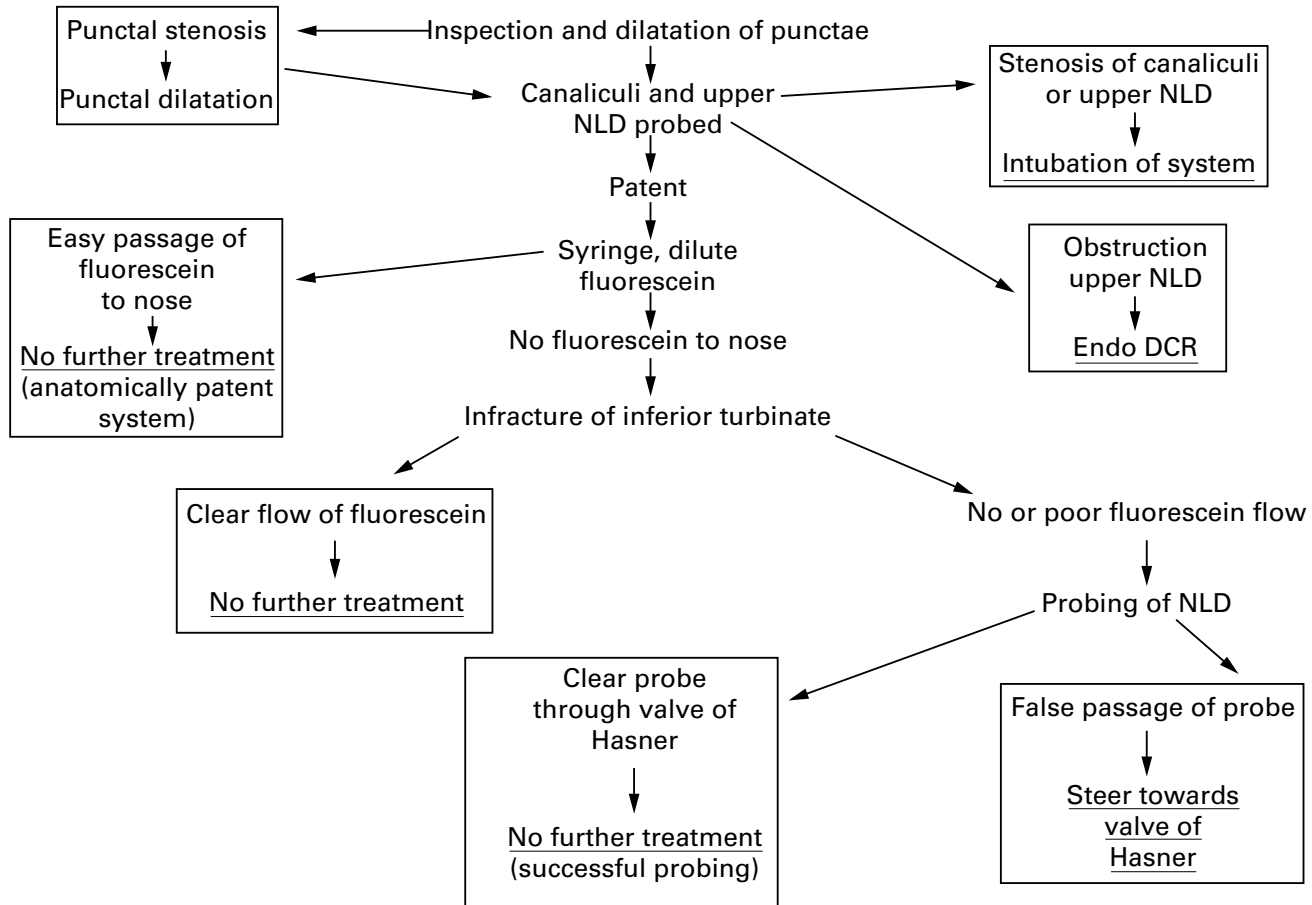

Figure 1 Flow diagram indicating the steps taken in probing.

punctal stenosis or other abnormality. Punctal stenosis or presence of a punctal membrane was treated with punctal dilatation or a punctal slit procedure. The puncta were dilated and each canaliculus gently probed as far as the lacrimal sac. Canalicular stenosis or tight partial obstruction of the upper end of the nasolacrimal duct was considered as an indication for intubation of the system with silicone tubes. Otherwise silicone tubes were not used.

Nasal endoscopy was performed while dilute fluorescein was injected via the upper canaliculus. If free flow of fluid was felt by the injector and observed in the inferior meatus then no further action was taken as the lacrimal outflow system was clearly anatomically patent and the symptoms were considered to be due to a "physiological" or functional blockage. Spontaneous resolution was awaited.

If free flow of fluorescein was not observed passing into the inferior meatus, or it was felt that the injector had to use increased pressure to effect a flow, then infracture of the inferior turbinate was performed. This procedure allows better visualisation of the distal end of the nasolacrimal duct but may also be curative in some cases as a result of stretching of the mucosal exit of the duct. ${ }^{24}$ The fluorescein flush was repeated while the distal end of the nasolacrimal duct was viewed directly through the nasal endoscope. If there was now free flow through the duct felt by the injector and seen by the endoscopist, then no further action was taken, as the inferior turbinate infracture was considered to have adequately opened the lower end of the nasolacrimal duct.

If there was complete obstruction (or atresia) or relative stenosis of the lower end of the nasolacrimal duct then a probing was performed. Atresia was diagnosed when no passage of fluorescein stained fluid was seen entering into the inferior meatus, although there was ballooning of the nasal mucosa due to the high pressure and the lack of patent mucosal orifice. Stenosis was diagnosed by the slow, often intermittent, passage of fluorescein into the nose (Fig 2) requiring high pressure by the injector. In both stenotic and atretic cases a probe was passed via the upper punctum and observed through the endoscope as it entered the inferior meatus. In the majority of cases the probe entered the inferior meatus via the valve of Hasner. This valve was identified as a small dimple in the mucosa of the lateral wall of the inferior meatus in atretic cases, or as the site of slow fluorescein entry into the nose in stenotic cases. Thus, if present, this permitted opening up the anatomically correct passage. If no valve was identified a cut down with a sickle knife onto the probe provided a large lower opening of the duct. This was done if the size of the mucosal perforation by the probe was insufficient to allow free flow of fluorescein stained fluid.

Two other methods of entering the inferior meatus were observed. (1) A false passage was formed with the probe entering the nose at a site which was disparate from the valve of Hasner, and (2) the probe was visualised in the submucosal space and continued its passage into the floor of the nose without actually perforating the mucosa and entering the nose (Fig 3). In both these situations the probe was steered, under endoscopic control, into the appropriate position.

If there was complete obstruction of the nasolacrimal duct an endoscopic DCR was performed instead of the probing under the same anaesthetic. 


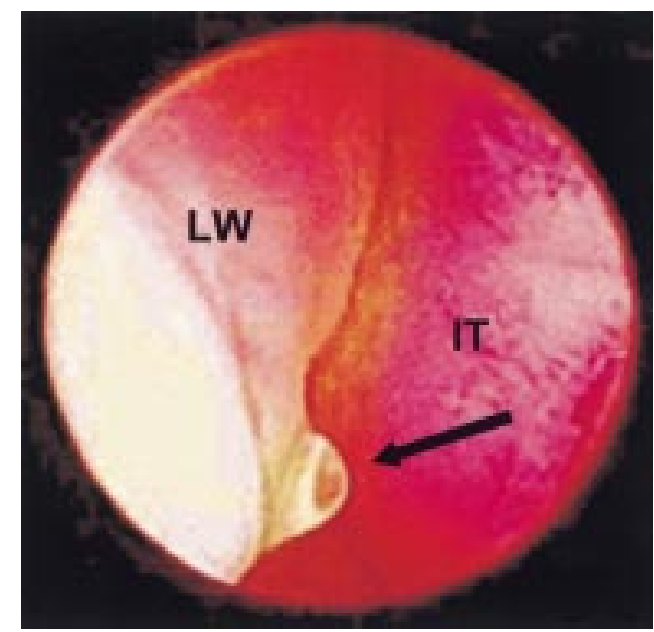

Figure 2 A bubble of fluorescein seen entering the nose in an intermittent manner in a case of stenosis of the distal end of the nasolacrimal. Arrow points to stenosed punctum. IT= inferior turbinate; $L W=$ lateral wall.

Patients were reviewed 1 month after the procedure and parents asked about the symptoms and a fluorescein disappearance test (FDT) was performed, ${ }^{22}$ to give an objective assessment of patency. Complete resolution, or a cure, was defined as a complete absence of watering or stickiness with a normal FDT. Partial resolution was diagnosed if the parents reported a complete, or near complete, remission of symptoms but with persistent relative delay of fluorescein clearance. Failure was defined as lack of any improvement in either the symptoms or the FDT.

\section{Results}

The study group comprised 40 children (52 nasolacrimal ducts) who underwent endonasal probing. The mean age at presentation was 32 months (10-89 months) and at probing was 34 months (range 12-91 months). There were 22 females and 18 males. There were 10 left, 18 right, and 12 bilateral cases. Three children had previously been probed in a conventional manner without success (one of these on two occasions).

Overall, the cure rate was $85 \%$, although the site and nature of the obstruction had a bearing

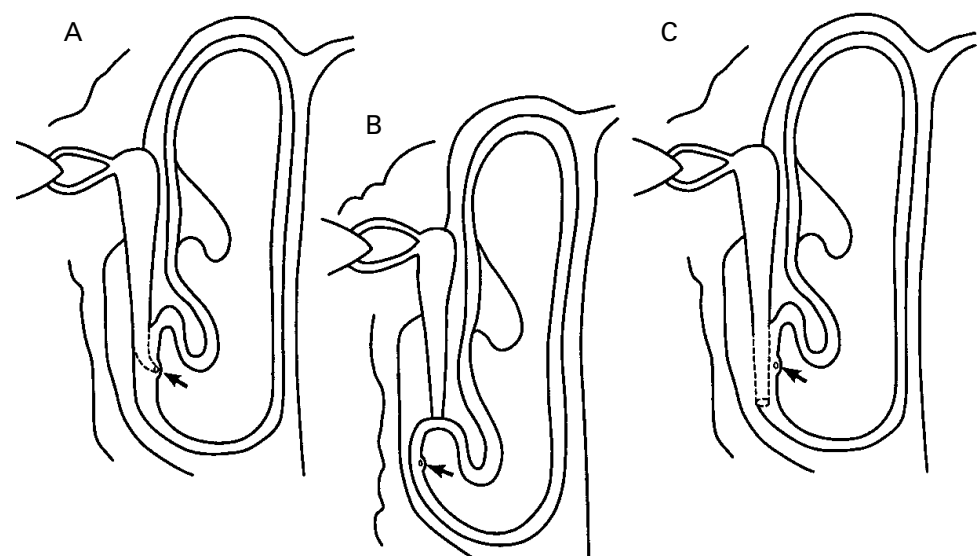

Figure 3 Diagrammatic representation of the possible methods that the nasolacrimal duct (NLD) may enter the nose. (A) Normal relation between osseous NLD and the mucosal exit. (B) The osseous NLD is shorter, with exit of the probe at the apex of the inferior meatus. (C) The osseous NLD is longer and the probe enters the submucosal space and passes to the floor of the nose, without entering the nose. on the outcome of the procedure. Four patients had evidence of anatomical patency, with a clear flow of fluorescein through the lacrimal system. A diagnosis of physiological blockage was made. Interestingly one of these children became asymptomatic after the syringing of the nasolacrimal duct, although the remaining three cases continued to have symptoms after the procedure as would be expected ( $75 \%$ failure rate). These cases tend to spontaneously improve with time - the reason for this being unclear, but presumably because of the development of a physiological lacrimal pump, although one case still has epiphora, at the age of 20 months, 6 months after the procedure.

A punctal membrane or stenosis was found to be the only identifiable cause of symptoms in six children. Treatment resulted in a cure in all cases $(100 \%)$. Five patients had a punctal anomaly in addition to other lacrimal abnormalities.

One patient had a common canalicular stenosis, diagnosed by a narrowing felt proximal to the sac when probed via both the upper and lower canaliculi. This was treated with dilatation of the area using a larger probe followed by the passage of silicone tubes. This resulted in a successful outcome.

Three children had a non-negotiable passage within the nasolacrimal canal providing an insurmountable blockage at the upper end of the nasolacrimal duct. In all three cases the procedure was extended to an endonasal DCR. In this group one patient was cured, one improved, and one was no better. Two had previously undergone probing.

Stenosis of the lower end of the nasolacrimal duct that was relieved by infracture of the inferior turbinate was present in six cases. This led to symptomatic relief in five cases $(83 \%)$. The one case that enjoyed only partial relief had been noted to have abnormal nasal mucosa in the inferior meatus but a free flow of fluid was observed after the infracture (topical steroids provided some degree improvement).

Fourteen cases had stenosis at the lower end of the NLD which required to be probed. In three cases the probe passed submucosally to the floor of the nose and had to be steered towards the valve of Hasner (Fig 3C). In one other case a false passage was made at the upper end of the inferior meatus (Fig 3B). Fluorescein had been seen entering the lateral wall of the meatus under high pressure through a stenotic valve before the probing. Fluorescein continued to pass through the stenotic valve rather than through the false passage. The probe was steered towards the valve of Hasner in a retrograde manner by the endoscopist as it was impossible to steer the probe through the valve from above. Every case of lower nasolacrimal duct stenosis was cured, although four cases had required a sickle knife cut down as the fistula made by the probe did not allow free passage of fluid.

There were six cases of lower nasolacrimal duct atresia. In one case the probe passed submucosally to the floor of the nose and required to be steered through the mucosa. In another case a false passage was made, which required 
reprobing via the "mucosal dimple". In none of the cases with atresia was the fistula made by the probe considered adequate, allowing a free flow of fluorescein, and a cut down was made in all cases. Five of the six cases (83\%) were cured.

There were no serious complications noted although some nasal bleeding occurred commonly. Overall, 33 (82.5\%) had complete resolution and two (5\%) partial resolution. Only one further procedure was required. In this instance a sickle knife cut down was made in a case that had atresia of the mucosal exit at the valve of Hasner. The result was the apparent free passage of fluorescein into the nose. In the follow up period there was no improvement in the symptoms and the FDT remained positive. No clear reason for the failure was identified and a repeat identical procedure achieved a cure. In all other cases the cause for failure was identified and no further treatment was undertaken.

\section{Discussion}

Congenital nasolacrimal duct obstruction is a condition which undergoes spontaneous resolution at the rate of approximately $95 \%$ of all affected individuals during the first year of life $\mathrm{e}^{5-12}$ and $60 \%$ of the remainder during the following year. ${ }^{1325}$ The results of probing during the second year of life are difficult to determine as reported studies are retrospective, not controlled, and are confounded by the continued high rate of spontaneous resolution. ${ }^{1426} 27$ In the only prospective, controlled trial into the effects of probing versus no treatment during the second year of life there was no significant difference in the outcome between probing and simple observation at 24 months of age. ${ }^{25}$ This study demonstrated that probing at 15 months of age is significantly better than observation, although by 24 months there was no difference between the two groups as cases of spontaneous resolution accumulated during the year. Probing, therefore, had the advantage that it cured the condition more rapidly but had the disadvantages that it required a surgical procedure under general anaesthetic, some cases of which would have spontaneously resolved in time, and that it runs the risk of iatrogenic damage to the outflow system.

It is well documented that the commonest cause of congenital nasolacrimal duct obstruction at birth is a membranous obstruction at the distal end of the nasolacrimal duct ${ }^{1-4}$; however, with a high rate of spontaneous resolution there is a natural selection process which ensures that increasingly complex problems become relatively more common as symptomatic children become older. Identifying the nature of these abnormalities is important as they may have a bearing on the optimum management and outcome. A major deficiency of previous reports is that the definition of probing failure is often vague and it remains unclear whether it is due to technical difficulties, the anatomical level of the obstruction, or the severity of the obstruction. We have attempted to address these problems in this paper by examining the possible reasons for probing failure in a group of children, using a structured technique combined with nasal endoscopy, which enables direct visualisation and precise identification of any abnormality in the area of the lower nasolacrimal duct.

When probing was performed with emphasis on diagnosing the underlying problem, a diversity of anomalies was found in these children who had had epiphora since birth which persisted beyond the age of 1 year. Twenty six out of the 40 children (65\%) had "classic" lower membranous obstruction in the form of either stenosis or atresia. Twenty four out of 25 $(96 \%)$ of these cases were cured with one probing procedure (excluding the case with abnormal nasal mucosa) and the one failure was cured with a second identical procedure. It is, however, worth noting that endoscopically guided relocation of the probe to prevent false passage formation was necessary in order to achieve this high degree of success. All six of the cases of isolated punctal anomaly were also cured, and it appears that if this is present as an isolated abnormality it carries a good prognosis. The cases that did not do well fell into two categories, those with anatomically patent lacrimal systems (functional blocks) and those with complete blockage of the upper nasolacrimal duct.

The anatomy of the nasolacrimal duct is variable and, rather than being one single passage, it may divide into several branches and enter the inferior meatus via more than one orifice. ${ }^{28}$ There may, however, be only one mucosal exit and this explains entry of the probe into the inferior meatus via a false passage. In addition, there may be disparity between the length of the osseous canal and the distal mucosal exit (the valve of Hasner) (Fig 3 ). If the nasolacrimal duct continues beyond the valve of Hasner then the probe may enter the submucosal space and a false passage is created down to the floor of the nose with no perforation of the mucosa into the nasal cavity, as seen in four cases (Fig 3C). A shorter duct entering the apex of the inferior meatus creates a false passage that does not reach the mucosal exit as seen in two cases (Fig 3B). It has already been recognised that some probing failures must be due to failure of mucosal perforation with creation of a false passage, ${ }^{29}{ }^{30}$ and this was clearly demonstrated by direct visualisation of the abnormal probe passage seen in our study.

The use of the sickle knife to perforate or increase the size of the mucosal fistula made by the probe is new. It is not possible to know, without controls, whether this part of the procedure is beneficial. It was, however, successful in producing a cure in those children where there was still evidence of stenotic flow, despite the passage of a probe into the inferior meatus or failure of the probe to penetrate the mucosa.

In six patients the primary cause of the obstruction was punctal stenosis or a punctal membrane which had not been diagnosed preoperatively despite attempted inspection of the area in the clinic using the slit lamp. Five out of six of these cases were bilateral and all were cured by opening or dilating the punctal 
orifices. In such cases it is important to complete an examination of the whole lacrimal system as a further six had lower nasolacrimal duct obstruction in addition to the punctal abnormalities (15\% therefore had pathology at each end of the lacrimal outflow system).

In those with physiological blockage endoscopy proved useful in that it confirmed no nasal abnormality such as close approximation of the inferior turbinate position to the nasal wall.

Endoscopically controlled infracture of the inferior turbinate may stretch the mucosal exit, and this procedure alone has been shown to increase the success rate of probing. ${ }^{24}$ In $15 \%$ of our patients infracture alone allowed a free flow of dilute fluorescein into the inferior meatus which had previously been blocked.

In a small number of cases no passage was successfully made into the nose, despite increasing the probe size. In these circumstances endoscopy is an advantage in that an endoscopic DCR can be undertaken under the same anaesthetic.

Our main purpose of adding nasal endoscopy to the standard technique of probing was to minimise the number of second procedures required. This was achieved in two ways: (1) by performing definitive surgery at the same time as the probing, and (2) by improving the understanding of the reasons for failure and thus preventing replication of a procedure which was doomed to failure again. In the 40 patients treated so far that target has been achieved with only one repeat procedure being required to date. Although not all cases have resolved completely, the reason for lack of success of the probing is more clearly understood and further unnecessary procedures were not undertaken. While follow up for some patients is limited and others have only partial resolution, there is good evidence that the severity of the residual lacrimal symptoms decreases with increasing age. ${ }^{31} 32$

In this study six (15\%) patients would almost certainly have had failed probings had endoscopy not been employed, as the probe either did not perforate the nasal mucosa at all or in a fashion that was non-functioning. This has emphasised that not all children with the signs and symptoms of CNLDO benefit from a simple probing, and that a clear knowledge of the nature of the pathology is essential in deciding both the optimum treatment and future management should the probing fail.

The incorporation of endoscopy increases the cost of the procedure that can only be justified if there is a clear improvement in the cure rate, with fewer procedures being required. Much larger numbers of patients will be required to decide this and hopefully other authors will employ the procedure and report full details of their patients to enable eventual meta-analysis. But in the meantime the use of this procedure has increased understanding of congenital nasolacrimal duct obstruction which, even if not used routinely, makes our knowledge of failures clearer.

1 Cassady JV. Developmental anatomy of the naso-lacrimal duct. Arch Ophthalmol 1952;47:141-58.

2 Grossman TH, Putz R. Uber die angeborene tranengangstenose der neugebornenen, ihre anatomie, ihre folgen und bahandlung. Klin Monatsbl Augenheilkd 1972;160:563-74.

3 Schwartz M. Congenital atresia of the naso-lacrimal canal. Arch Ophthalmol 1935;13:301-2.

4 Fiori-Ratti. In: Duke-Elder, ed. Textbook of ophthalmology. Vol 5; Chapter LVIII. Diseases of the lacrimal system. London: Kimpton, 1952

5 MacEwen CJ, Young JDH. Epiphora in the first year of life. Eye 1991;5:596-600

6 Ffookes OO. Dacryocystitis in infancy. Br $\mathcal{F}$ Ophthalmol 1962;46:422-34.

7 Guerry D, Kendig EL. Congenital impatency of the naso-lacrimal duct. Arch Ophthalmol 1948;39:193-204.

8 Cassady JV. Dacryocystitis of infancy. Am $\mathcal{f}$ Ophthalmol 1948;31:773-80.

9 Noda S, Hayasaka S, Tomoichi S. Congenital naso-lacrimal duct obstruction in Japanese infants:it's incidence and treatment with massage. $\mathcal{F}$ Pediatr Ophthalmol Strabismus 1991;28:20-2.

10 Korchmaros I, Szalay E, Fodor M, et al. Probable aetiology of the non-opened lacrimal pathway. In: Yamaguchi A, ed. Recent advances in the lacrimal system. 1981.

11 Kendig EL, Guerry D. The incidence of congenital impatency of naso-lacrimal duct obstruction. $\mathcal{F}$ Pediatr 1950;36:212-3

12 Murako del castillo J. Development of the lacrimal apparatus. In: Milder B, Weil B, eds. The lacrimal system. Morwalk, CT: Appleton-Century-Crofts, 1983:9-22.

13 Price HW. Dacryostenosis. F Pediatr 1947;30:302-5.

14 Katowitz JA, Welsh MG. Timing of initial probing and irrigation in congenital naso-lacrimal duct obstruction. gation in congenital naso-lacrim
Ophthalmology 1987;94:698-705.

15 Paul TO. Medical management of congenital naso-lacrimal duct obstruction. F Pediatr Ophthalmol Strabismus 1985;22: duct obs-70.

16 Peterson RA, Robb RM. The course of congenital obstruction of the naso-lacrimal duct. F Pediatr Ophthalmol Strabismus 1978;15:246-50.

17 Nelson LB Calhoun JH, Menduke H. Medical management of congenital naso-lacrimal duct obstruction. Ophthalmology 1985;92:1187-90.

18 Nucci P, Capoferri C, Alfarano R, et al. Conservative management of congenital naso-lacrimal duct obstruction. $\mathcal{F}$ Paediatr Ophthalmol Strabismus 1989;26:39-43.

19 Wobig JL. Lacrimal probing complications. Ophthalmic Plast Reconstr Surg 2985;1:75-6.

20 Milder B. Complications in lacrimal surgery. In: Waltman SR, Krupin T, eds. Complications in ophthalmic surgery. New SR, Krupin T, eds. Complications in

21 Paul TO, Shepherd R. Congenital naso-lacrimal duct obstruction:natural history and the timing of optimal intervention. F Pediatr Ophthalmol Strabismus 1994;31:362-7.

22 MacEwen CJ, Young JDH. The fluorescein disappearance test: an evaluation of its use in infants. F Pediatr Ophthalmol Strabismus 1991;28:302-5.

23 Ram B, Barras CW, White PS, et al. The technique of nasendoscopy in the evaluation of naso-lacrimal duct obstruction in children. Rhinology 2000;38:83-6.

24 Wesley RE. Inferior turbinate fracture in the treatment of congenital naso-lacrimal duct obstruction and congenital naso-lacrimal duct anomaly. Ophthalmic Surg 1985;16: 368-71.

25 Young JDH, MacEwen CJ, Ogsten S. Congenital nasolacrimal duct obstruction in the second year of life: a multicentre trial of management. Eye 1996;10:485-91.

26 Robb RM. Success rates of naso-lacrimal duct probing at time intervals after 1 year of age. Ophthalmology 1998;105: time inter $1307-10$.

27 El Mansoury J, Calhoun JH, Nelson LB, et al. Results of late probing for congenital naso-lacrimal duct obstructrion. Ophthalmology 1986;93:1052-4.

28 Boger WP. Congenital naso-lacrimal duct obstruction. In: Albert DM, Jakobiec FA, eds. Principles and practice of ophthalmology, clinical practice. Philadelphia: WB Saunders, 1994:2812-26.

29 Ingels K, Kestelyn P, Meire F, et al. The endoscopic approach for congenital naso-lacrimal duct obstructrion. Clin Otolaryngol 1997;22:96-9.

30 Mirecki R. Cause of failure in probing the naso-lacrimal duct in infants and children and ways of avoiding them. $f$ Paediatr Ophthalmol 1968;5:171-5.

31 Sturrock SM, MacEwen CJ, Young JDH. Long term results after probing for congenital naso-lacrimal duct obstrucafter probing for congenital naso-lactin
trion. Br f Ophthalmol 1994;78:892-4

32 Maini R, MacEwen CJ, Young JDH. The long-term effects of congenital naso-lacrimal duct obstruction. Eye 1998;12: 669-71. 